\title{
Lattice BGK Simulations of Unsteady Flow in a 2D Elastic Tube
}

\author{
Alfons G. Hoekstra, Jos van ‘t Hoff, Abdel Monim M. Artoli, and Peter M.A. Sloot
}

\author{
Section Computational Science \\ Faculty of Science, University of Amsterdam \\ http: //www. science.uva.nl/research/scs \\ alfons@science.uva.nl
}

\begin{abstract}
We report results of unsteady, harmonic flow simulations with the lattice BGK method in two-dimensional elastic tubes. The tubes are assumed to obey a simple constitutive equation, linearly relating the diameter of the tube to the pressure difference inside and outside the tube. First, as a benchmark, we present results of steady flow in such elastic tubes, and compare the performance of three different boundary conditions for the solid wall. Next, we present results of unsteady (harmonic) flow in the elastic tube, and validate the results by comparing them with theoretical expressions for the dispersion relation of the complex speed of traveling waves in the tube. Within the range of Womersley numbers tested the agreement between the simulations and the theory is good.
\end{abstract}

\section{Introduction}

We have a strong interest in simulating unsteady flow in complex geometry using the Lattice Boltzmann BGK method (LBGK, for an introduction we refer to [1]). In particular, we focus on modeling blood flow in the large arteries of the human body. We recently showed that LBGK is in principle capable of simulating such flows within the range of Reynolds - and Womersley numbers that exist in the large arteries [2]. As a proof of concept we applied LBGK to simulate flow in the lower abdominal aorta, including the bifurcation to the left and right illiac arteries (supplying the legs with blood) $[3,4]$.

In those simulations we assume that 1) blood is a Newtonian fluid and 2) that the arteries are rigid structures. The first assumption is generally acknowledged to be valid in the large arteries [5-7]. This is because of the relative high shear stress in those arteries that renders a constant viscosity of 4 centipoise for whole blood. Despite this a number of groups do investigate the influence of the true non-Newtonian nature of blood on the flow profiles and shear stresses in the arteries [see e.g. 8,9]. As to the second assumption, arteries as rigid structures, it is well known that, due to the pulsatile nature of blood flow and the elasticity of the arteries and surrounding tissue, during a systolic cycle the 
diameter of the larger arteries may vary 5-10\% (for a discussion, see e.g [10]). However, in typical hemodynamic simulations the computational grid is obtained from Magnetic Resonance Imaging (MRI) of real arteries. Such images obtained through MRI typically have errors in the position of the artery lumen (i.e. the artery wall) of 1-8 \% [11]. Therefore, given this accuracy, the influence of elasticity of the wall can be considered a secondary effect. However, simulations do suggest that the movement of the wall may have influence on the resulting flow fields, and more important in vascular disease, on the resulting shear stress distributions on the wall [e.g. 7,12]. As we are currently working on developing a virtual operating theatre for vascular reconstruction $[13,14]$ the issue of wall elasticity may be of importance. We therefore aim to relax this rigid wall assumption and include elasticity of the arteries into our LBGK simulations.

In this paper we set the first steps towards including elastic walls into the LBGK model. We will study the case of two-dimensional harmonic flow in a long and slender elastic tube. This was previously done by Fang et al. [15]. They developed a tailored solid boundary condition to be used for moving walls, and studied the case of steady flow and of a harmonic flow. By comparing with theoretical expressions for steady flow they could demonstrate the validity of their approach. For the harmonic flow they solely presented simulation results. In this paper we extend the results of Fang et al. in two ways. First, we compare the performance of three different boundary conditions in the case of a steady flow in an elastic tube. Next, we perform unsteady harmonic flow simulations and compare the results with theory, especially with a dispersion relation for the complex speed of sound of the travelling waves in the tube.

\section{Theory}

The topic of unsteady flow in elastic tubes has a long history (see e.g. [5]), with a first complete theoretical description for long and slender tubes being formulated by Womersley [16]. Since then, this theory has been refined and extended in many respects (for an overview, see e.g. [17]). However, we will use Womersley's theory to validate our simulations.

We assume a linear pressure-radius relationship:

$$
R=R_{0}+\beta p / 2
$$

$p$ is the transmural pressure (i.e. the difference between the inside and outside pressure), $R_{0}$ the radius for zero transmural pressure and $\beta$ a compliance constant. This relationship is a good representation for the large arteries. [5]

First consider a steady flow driven by a pressure gradient. Because of the pressure drop the radius at the high-pressure end will be larger than at the low-pressure end. We assume that the tube is long and slender, that the flow is laminar and one-dimensional, and that the entry - and exit effects are neglected. In that case the local flow field can be assumed to be the well-known parabolic Poiseuille profile. Using this assumption in combination 
with Eq. 1 and the fact that the flow rate $Q$ in every cross section of the tube must be constant, immediately leads to the following expression ${ }^{1}$ : [5]

$$
3 v \beta Q z=[R(0)]^{4}-[R(z)]^{4},
$$

with $v$ the viscosity of the fluid and $R(z)$ the radius of the tube at a distance $z$ from the inlet. By substituting the tube length $L$ in Eq. 2 we get an expression for $3 v \beta Q z$ in terms of the inlet - and outlet radius. Substituting that expression back into Eq. 2 yields an explicit expression for $R(z)$ in terms of the inlet - and outlet radius:

$$
R(z)=\left\{\left([R(L)]^{4}-[R(0)]^{4}\right) \frac{z}{L}+[R(0)]^{4}\right\}^{1 / 4} .
$$

Finally, by substituting Eq. 3 into Eq. 1 we obtain an expression for the pressure in the tube. Using the expression for the Reynolds number for Poiseuille flow we can now immediately derive

$$
\operatorname{Re}=\frac{[R(0)]^{4}-[R(L)]^{4}}{2 \beta L v^{2}}
$$

The analytical solution for time dependent flow in an elastic tube was first described by Womersley [16]. Here we just provide an outline of his theory. For all details we refer to the original paper. The theory assumes that the non-linear terms in the Navier-Stokes equations may be ignored (i.e. small Reynolds number flows). The tube is assumed to be a thin-walled elastic tube with radius $R$, wall thickness $h$, density of the tube wall $\rho$. Moreover, the density of the fluid is $\rho_{0}$. The equations for the displacement of the wall and the fluid motion are coupled through a set a boundary conditions at the inner wall of the tube. Introducing a speed of sound $c$ and looking at the type of motion in which $u / c$, $v / c$, and $\omega R / c$ are small (with $u$ and $v$ the longitudinal - and radial components of the fluid velocity, and $\omega$ the angular frequency of the driving harmonic pressure gradient), we can write

$$
\begin{gathered}
p=p_{1} \exp [i \omega(t-z / c)], \\
u=u_{1} \exp [i \omega(t-z / c)], \\
v=v_{1} \exp [i \omega(t-z / c)] .
\end{gathered}
$$

Next, the equations of motion can be solved and in combination with the boundary conditions four homogeneous linear equations in four unknowns are obtained. A non trivial solution is only possible by requiring that the determinant of this system of equations is zero, thus resulting in a dispersion relation which will determine the wave

\footnotetext{
${ }^{1}$ Note that Eq. 2 differs from the expressions in Ref. [5] because we consider two-dimensional flows here.
} 
1000 A.G. Hoekstra et al.

velocity $c$ in terms of the elastic properties of the tube and $\alpha$, the non-dimensional Womersley number $(\alpha=R \sqrt{\omega / v})$. The final dispersion relation reads

$$
\left(1-\sigma^{2}\right)(1-F) x^{2}-x\left[2+k(1-F)+F\left(\frac{1}{2}-2 \sigma\right)\right]+F+2 k=0,
$$

where

$$
\begin{gathered}
F=2 \frac{J_{1}\left(\alpha i^{3 / 2}\right)}{\alpha i^{3 / 2} J_{0}\left(\alpha i^{3 / 2}\right)}, \\
k=\frac{h \rho}{R \rho_{0}}, \\
x=\frac{2}{\left(1-\sigma^{2}\right)} \frac{c_{0}^{2}}{c^{2}},
\end{gathered}
$$

with $\sigma$ Poisson's ratio of the elastic material of the tube, $J_{0}$ and $J_{1}$ the zeroth and first order Bessel functions, and $c_{0}$ the speed of sound in the 'empty' tube. By solving Eq. 6 we find $\mathrm{a}$, in general complex valued expression for $x$. If we now write

$$
\frac{c_{0}}{c}=\sqrt{\left(1-\sigma^{2}\right) \times / 2}=X-i Y
$$

and substitute this into Eq. 5 we immediately recognize that the wave velocity $c_{1}$ of the pressure waves in the tube becomes

$$
c_{1}=\frac{c_{0}}{X}
$$

and that the wave after travelling over a distance of one wavelength is attenuated by a factor $\gamma$,

$$
\gamma=\exp \left[-2 \pi \frac{Y}{X}\right]
$$




\section{Results}

\subsection{Description of the Simulations}

For the fluid flow simulations we apply the D2Q9 Lattice BGK model [1]. For the solid walls we have tested three different boundary conditions. The Velocity Boundary Condition (VBC) by Zou and He [18], the recently proposed boundary condition by Bouzidi et al. [19] and another recently proposed boundary condition by Fang et al. [15]. The last one was specifically developed with moving boundaries in mind. At the inlet and outlet we use pressure boundary conditions. For steady flows a constant pressure difference is prescribed. For the unsteady flow the density at the inlet was set to $1+$ $0.001 \times \cos (2 \pi t / T)$ and at the outlet to 1 . Here, $t$ denotes time and $T$ the period of oscillation (both measured in units of $\Delta t=1$ ).

As the solid walls move during the simulation, it may happen that fluid nodes turn into boundary nodes and boundary nodes into solid nodes, or the other way around. If a boundary node turns into a fluid node we need some prescription to assign values to the yet unknown distribution function on the new fluid nodes. For this we use the method described by Fang et al. [15], where the unknown distribution functions are assigned through second order extrapolation in the directions that pointed towards fluid nodes in the previous time step.
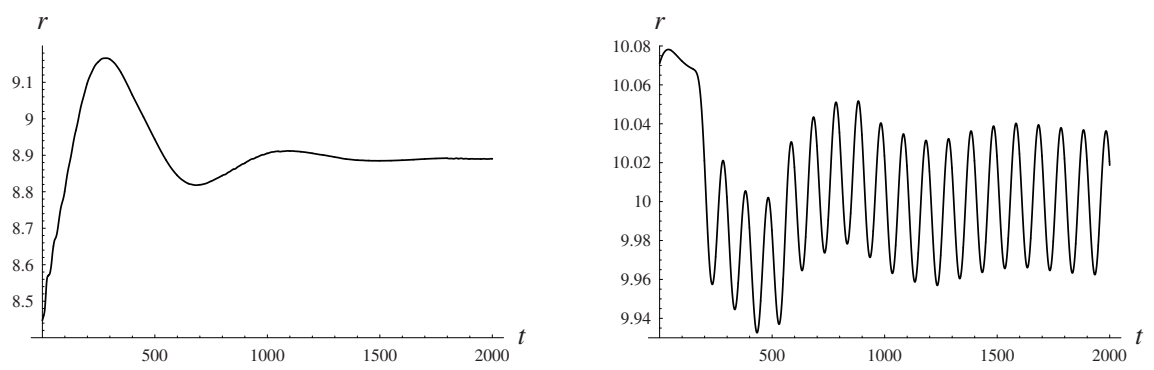

Fig. 1. Settling of the radius (vertical axis) at $\mathrm{z}=\mathrm{L} / 2$ in the 2000 time steps (horizontal axis) after the walls are released. Left panel: steady flow on a $20 \times 100$ lattice $(\operatorname{Re}=1)$; right panel: harmonic flow on a $10 \times 100$ lattice with $\mathrm{T}=100$.

The coupling between the fluid and the elastic wall is through Eq. [1]. We first start a simulation with a fixed wall and allow the flow field to reach a steady state. Next, the wall is released. In every cross section of the tube the pressure is measured, and the radius is instantaneously adapted to the value given by Eq. [1]. Next, a LBGK iteration is performed, the pressure is again measured, etc. This cycle is iterated until the tube has settled (in case of steady flow) or performs steady harmonic oscillations (in case of the 
harmonic flow). Figure 1 shows an example of this settling of the tube wall for both steady and period flows. For harmonic flow in tubes with a smaller inlet diameter the convergence is faster (data not shown). The reason for not starting the simulation immediately with elastic walls is that the stability of the simulations enhances significantly if the flow is first allowed to settle in the uniform rigid tube (data not shown).

\subsection{Steady Flow}

We have performed a set of simulations where the length of the tube was 600 , the inlet diameter was set to 30 and the outlet diameter to 21 . The Renolds number was set to 1 and 10 respectively. First we show, in Fig. 2, the resulting diameter as a function of position in the tube for the three boundary conditions. The theoretical curve (Eq. 3) is shown as well. For other choices of diameters the results are comparable. We calculate an average error defined by

$$
\varepsilon_{\text {wall }}=\frac{\sum\left|R_{T}(z)-R_{S}(z)\right|}{\sum\left|R_{T}(z)\right|}
$$

where the subscript $T$ denotes the theory and $S$ the simulation. Table 1 shows the resulting errors.

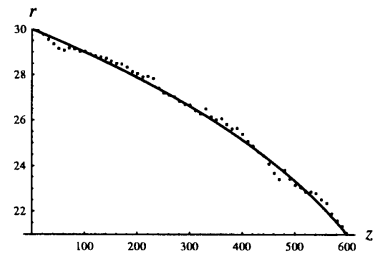

(a)

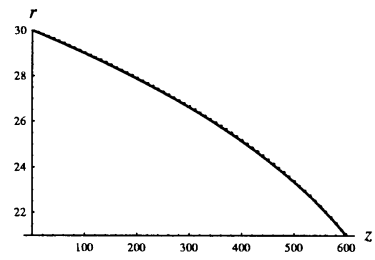

(b)

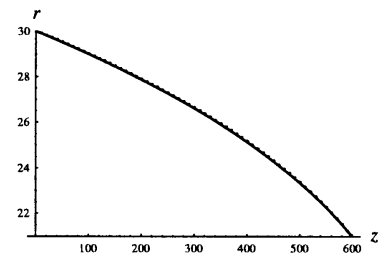

(c)

Fig. 2. Theoretical shape of the upper wall of an elastic tube due to steady flow compared to the simulated results of the Bouzidi- (a), Zou- (b) and Fang-boundary (c) at $\operatorname{Re}=10$.

Table 1. Relative errors in the shape of an elastic tube at several Reynolds numbers

\begin{tabular}{|l|c|c|}
\hline \multicolumn{1}{|c|}{ Bound. condition } & $\boldsymbol{R e}=\mathbf{1}$ & $\boldsymbol{R e}=\mathbf{1 0}$ \\
\hline Fang & 0.0006 & 0.0014 \\
\hline Bouzidi & 0.0063 & 0.0059 \\
\hline Zou & 0.0006 & 0.0013 \\
\hline
\end{tabular}

We have also measured the error in the resulting velocity fields (using the same error definition as in Eq. 11), as a function of the grid spacing. The results are presented in Fig. 
3. The error behavior is first order in the grid spacing (to be precise, for $\mathrm{Re}=1$ the error goes as $\Delta x^{1.4}$ for all three boundary conditions, and for $\operatorname{Re}=10$ as $\Delta x^{1.4}$ for the Bouzidi boundary condition and as $\Delta x^{1.1}$ for the other two).
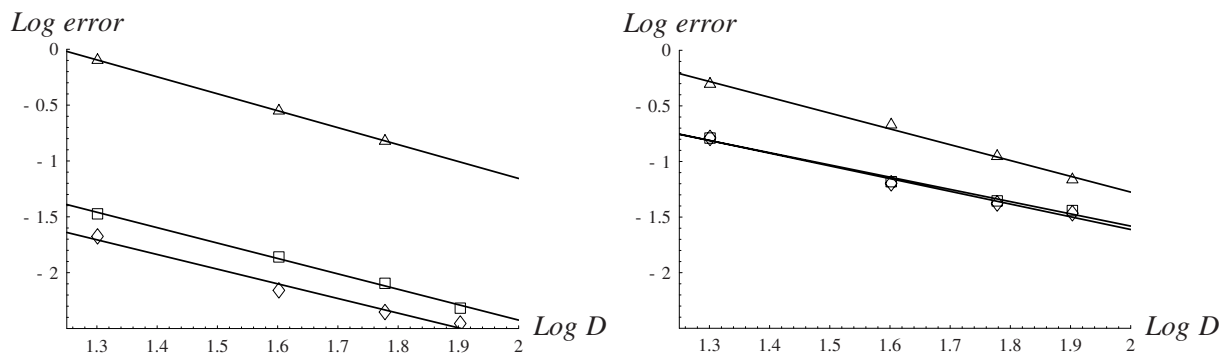

Fig. 3. Relative errors in velocity profiles as a function of the grid spacing for the Fang (diamond), Bouzidi (box) and Zou (triangle) boundary conditions at Reynolds numbers 1 (left) and 10 (right). Solid lines are the linear fits.

\subsection{Unsteady Flow}

For the unsteady flow simulations we only applied the Fang boundary. In Fig. 4 we show the propagating pressure wave that is induced in the elastic tube. Comparing the results of the simulations to the dispersion relation in Eq. 6 is done by analyzing these pressure waves. The only parameter that was changed for different simulations is the radius of the tube under zero transmural pressure. With the radius, the Womersley number also changes. This has an effect on the attenuation of the amplitude of the pressure wave (through the function $F$, see Eq. 7.a). For large $\alpha$ the amplitude decreases less per wavelength than for small $\alpha$.

If we put the Poisson ratio and the thickness of the wall to zero in Eq. 6, which is the case for our simulations, we find for the attenuation constant $\gamma$ the following theoretical values: at $\alpha=3.07$ an attenuation constant of 0.102 ; if we then increase the radius by a factor 2, which increases $\alpha$ to 6.14, we find an attenuation constant of 0.409 . This means the amplitude of the pressure wave drops faster along the length of the tube for lower Womersley number. The attenuation constant $\gamma$ is obtained from the simulations by fitting the simulated pressure waves with the theoretical expressions. The results, for a range of Womersley parameters, are shown in Fig. 5 and Table 2. 


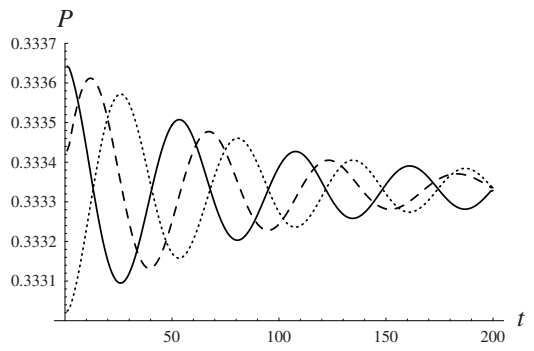

Fig. 4. Propagation of the pressure wave. The pressure distributions at $\mathrm{t}=20 \mathrm{~T}$ (solid line), $20.25 \mathrm{~T}$ (dashed line) and $20.5 \mathrm{~T}$ (dotted line) are shown for a tube of length 200 and $\mathrm{R}=10$. ( $\mathrm{T}=100$ and $\alpha=6.14$ ).

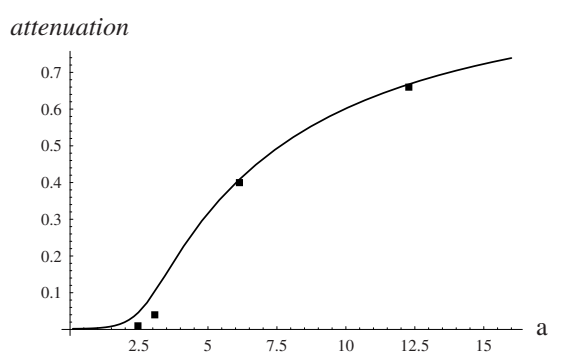

Fig. 5. Simulated (box) and theoretical (line) attenuation constants as a function of the Womersley number. The line represents the theory $(\sigma=0, \mathrm{k}=0)$.

Table 2. Numerical values of the theoretical and simulated attenuation constants presented in Fig. 5

\begin{tabular}{|c|c|c|}
\hline $\boldsymbol{\alpha}$ & Theoretical att. constant & Simulated att. constant \\
\hline 2.46 & 0.04 & 0.01 \\
3.07 & 0.10 & 0.04 \\
\hline 6.14 & 0.41 & 0.40 \\
\hline 12.28 & 0.67 & 0.66 \\
\hline
\end{tabular}

For larger Womersley numbers the agreement between simulation and theory is perfect. However, for the small $\alpha$ values we observe a large difference. This discrepancy may be attributed to discretization errors. For instance, for $\alpha=2.46$ the radius of the inlet was only 5 grid points. We performed another simulation for the same $\alpha$ but now with a radius of 20 grid points (and a tube length of 3500 and $T=2500$, thus dramatically increasing execution times to 80 hours on a $300 \mathrm{MHz}$ Sun workstation). After fitting we now obtained $\gamma=0.031$. This result is much closer to the theory, but still the error is relatively large.

The influence from the compliance constant (i.e. the elasticity) of the wall on the pressure waves comes only through $c_{0}$, the wave velocity for a non-viscous fluid. ${ }^{2}$ In other words, we expect that the attenuation is not influenced by the compliance constant. To check this we ran a simulation for $\alpha=6.14$ with a twice as large compliance constant. In that case we found $\gamma=0.43$.

We have also measured the wavelength of the pressure waves $\lambda=c_{0} T / X$. Unfortunately, because of our specific simulation setup a theoretical expression for $c_{0}$ was not immediately obvious. Therefore, we have looked at ratios of measured wavelengths at

2 And of course also through the Poisson's ratio, but here we have taken $\sigma=0$. 
different Womersley numbers, which only depend on $X(\alpha)$. The results are shown in Table 3.

Table 3. Theoretical and simulated ratios between the wavelengths at different Womersley numbers

\begin{tabular}{|c|c|c|c|}
\hline$\alpha_{1}$ & $\alpha_{2}$ & $\lambda\left(\alpha_{1}\right) / \lambda\left(\alpha_{2}\right)$ Theory & $\lambda\left(\alpha_{1}\right) / \lambda\left(\alpha_{2}\right)$ Simulated \\
\hline 2.46 & 3.07 & 0.94 & $0.98 \pm 0.07$ \\
\hline 2.46 & 6.14 & 0.86 & $0.95 \pm 0.07$ \\
\hline 2.46 & 12.28 & 0.82 & $0.93 \pm 0.07$ \\
\hline 3.07 & 6.14 & 0.92 & $0.96 \pm 0.07$ \\
\hline 3.07 & 12.28 & 0.88 & $0.95 \pm 0.07$ \\
\hline 6.14 & 12.28 & 0.95 & $0.98 \pm 0.07$ \\
\hline
\end{tabular}

\section{Discussion}

We presented a first set of results of LBGK simulations with elastic walls. From our steady flow benchmarks we observe that the Fang boundary and Zou boundary have comparable error behavior. Notice that in combination with moving walls the error in the velocity is first order in the grid spacing. The Bouzidi boundary also is first order, but has a much larger value of the errors. For non-moving walls this boundary condition is very good (see e.g. 2). However, in combination with elastic walls our results suggest that the details of the interpolations result wrong a density (and therefore pressure) field close to the wall. This has an immediate effect on the location of the walls, see Eq. 1 and the resulting velocity fields.

In the simulations for unsteady flow we have used the Fang boundary. For a rigid tube we were able to perfectly reproduce Womersley flow with this boundary condition (data not shown). For the elastic wall the results are very promising. We reproduce the attenuated pressure waves as predicted by theory. Moreover, over a range of Womersley numbers we have demonstrated a good agreement between the theoretical dispersion relation for the complex wave velocity and our simulations. The relative large errors for small Womersley number need further investigation, but the suggested discretization effect may play an important role.

The elastic wall in our simulations was highly simplified: it was mass-less and had a zero thickness. This is not very realistic, and as a next step we will consider tubes with a thin wall and nonzero mass. This means that we will also have to integrate the equations of motion of the wall. We will consider other constitutive equations for the wall, such as a linear material described by a Young's modulus and a Poisson ratio. In this case the $c_{0}$ is given by the Moens-Korteweg formula [5]. This will allow a more extensive validation of our simulations, by comparison with Eq. 6 over a large range of parameter values. Moreover, we will also extend the simulations to three dimensions, and consider more realistic non-symmetrical cases. 


\section{References}

1. Succi, S.: The Lattice Boltzmann Equation for Fluid Dynamics and Beyond. Oxford University Press, Oxford (2001)

2. Artoli, A.M., Hoekstra, A.G., and Sloot, P.M.A.: 3D Pulsatile Flow in the lattice Boltzmann BGK Method. Int. J. Mod. Phys. C 13 (2002) 1119-1134

3. Artoli, A.M., Hoekstra, A.G., and Sloot, P.M.A.: Simulation of a Systolic Cycle in a Realistic Artery with the Lattice Boltzmann BGK Method. submitted to Int. J. Mod. Phys. B. (2002)

4. Artoli, A.M., Hoekstra, A.G., and Sloot, P.M.A.: Mesoscopic Simulations of Flow in the Human Abdominal Artery. submitted to J. Biomech. (2002)

5. Fung, Y.C.: Biomechanics, Circulation. Springer, New York Berlin Heidelberg (1984)

6. Ku, D.N.: Blood Flow in Arteries. Annu. Rev. Fluid Mech. 29 (1997) 399-434

7. Vorp, D.A., Steinman, D.A., and Ethier, C.R.: Computational Modeling of Arterial Biomechanics. IEEE Comput. Sci. Eng. 3 (September/October 2001) 51-64

8. Gijsen, F.J.H., van de Vosse, F.N. and Janssen, J.D.: The Influence of the non-Newtonian Properties of Blood on the Flow in Large Arteries: Steady Flow in a Carotid Bifurcation Model. J. Biomech. 32 (1999) 601-608

9. Gijsen, F.J.H., Allanic, E., van de Vosse, F.N. and Janssen, J.D.: The Influence of the nonNewtonian Properties of Blood on the Flow in Large Arteries: Unsteady Flow in a $90^{\circ}$ Curved Tube. J. Biomech. 32 (1999) 705-713

10. Taylor, C.A., Hughes, T.J.R., and Zarins, C.K.: Finite Element Modeling of Three-Dimensional Pulsatile Flow in the Abdominal Aorta: Relevance to Atherosclerosis. Annals of Biomed. Eng. 26 (1998) 975-987

11. Moore, J.A., Steinman, D.A., and Ethier, C.R.: Computational Blood Flow Modeling: Errors Associated with Reconstructing Finite Element Models from Magnetic Resonance Images. J. Biomech. 31 (1998) 179-184

12 Perktold, K. and Rappitsch, G.: Computer Simulations of Local Blood Flow and Vessel Mechanics in a Compliant Carotid Artery Bifurcation Model. J. Biomech. 28 (1995) 845-856

13. Belleman, R.G. and Sloot, P.M.A.: The Design of Dynamic Exploration Environments for Computational Steering Simulations. In: Bubak; M., Mošcinski, J. and Noga, M. (eds.): Proceedings of the SGI Users' Conference 2000. ISBN 83-902363-9-7. (2000) 57-74

14. Belleman, R.G. and Sloot, P.M.A.: Simulated vascular reconstruction in a virtual operating theatre. In Lemke, H.U., Vannier, M.W., Inamura, K., Farman, A.G., and Doi, K. (eds.): Computer Assisted Radiology and Surgery. Excerpta Medica, International Congress Series 1230 (2001) 938-944

15. Fang, H., Wang, Z., Lin, Z., and Liu, M.: Lattice Boltzmann Method for Simulating the Viscous Flow in Large Distensible Arteries. Phys. Rev. E 65 (2002) 051925

16. Womersley, J.R.: Oscillatory Motion of a Viscous Liquid in a Thin-Walled Elastic Tube - I: The Linear Aproximation for Long Waves. Phil. Mag. 46 (1955) 199-221

17. Sramek, B.B., Valenta, J., and Klimes, F (eds.): Biomechanics of the Cardio-Vascular System. Czech Technical University Press, Prague (1995)

18. Zou, Q., and He, X.: On Pressure and Velocity Boundary Conditions for the Lattice Boltzmann BGK Model. Phys. Fluids 9 (1997) 1591

19. Bouzidi, M., Firdaouss, M., and Lallemand, P.: Momentum Transfer of a Lattice Boltzmann Fluid with Boundaries. Phys. Fluids 13 (2001) 3452-3459 